Proceedings of the Edinburgh Mathematical Society (2002) 45, 631-645 (C)

DOI:10.1017/S0013091501000773 Printed in the United Kingdom

\title{
STURM-LIOUVILLE PROBLEMS WITH BOUNDARY CONDITIONS RATIONALLY DEPENDENT ON THE EIGENPARAMETER. I
}

\author{
PAUL A. BINDING ${ }^{1}$, PATRICK J. BROWNE ${ }^{2}$ AND BRUCE A. WATSON ${ }^{3}$ \\ ${ }^{1}$ Department of Mathematics and Statistics, University of Calgary, \\ Calgary, Alberta, Canada T2N 1N4 (binding@ucalgary.ca) \\ ${ }^{2}$ Mathematical Sciences Group, Department of Computer Science, \\ University of Saskatchewan, Saskatoon, Saskatchewan, \\ Canada S7N 5E6 (browne@admin.usask.ca) \\ ${ }^{3}$ Department of Mathematics, University of the Witwatersrand, Private Bag 3, \\ PO WITS 2050, South Africa (watson@maths.wits.ac.za)
}

(Received 22 August 2001)

Abstract We consider the Sturm-Liouville equation

$$
-y^{\prime \prime}+q y=\lambda y \quad \text { on }[0,1]
$$

subject to the boundary conditions

$$
y(0) \cos \alpha=y^{\prime}(0) \sin \alpha, \quad \alpha \in[0, \pi),
$$

and

$$
\frac{y^{\prime}}{y}(1)=a \lambda+b-\sum_{k=1}^{N} \frac{b_{k}}{\lambda-c_{k}} .
$$

Topics treated include existence and asymptotics of eigenvalues, oscillation of eigenfunctions, and transformations between such problems.

Keywords: Sturm-Liouville; eigenparameter-dependent boundary condition; eigenfunction oscillation; eigenvalue asymptotics

AMS 2000 Mathematics subject classification: Primary 34B24; 34L20

\section{Introduction}

We consider the regular Sturm-Liouville equation

$$
-y^{\prime \prime}+q y=\lambda y \quad \text { on }[0,1]
$$

subject to the boundary conditions

$$
y(0) \cos \alpha=y^{\prime}(0) \sin \alpha, \quad \alpha \in[0, \pi),
$$

and

$$
\left(y^{\prime} / y\right)(1)=f(\lambda)
$$


for a class of functions $f$. The case when $f$ is constant is the 'standard' Sturm-Liouville problem on which there is a vast literature. Cases where $f$ is affine or bilinear make up the majority of the literature on so-called 'eigenvalue-dependent boundary conditions' and we refer to $[\mathbf{1 4 , 2 6}]$ and the many references cited therein for some of this activity. Alternative settings have also been studied: for example, see $[\mathbf{1 5}-\mathbf{1 7}]$ for singular equations; $[\mathbf{9}, \mathbf{1 2}, \mathbf{2 2}]$ for higher-order (and matrix) equations; and $[\mathbf{5}, \mathbf{1 9}, \mathbf{2 7}]$ for partial differential equations. There have also been several investigations of (1.1)-(1.3) where $f$ is a more general (usually rational) function (see, for example, $[\mathbf{3}, \mathbf{2 1}, \mathbf{2 3}, \mathbf{2 5}]$ and references cited therein).

Our study is the first of two parts on problems involving a particular class $\mathcal{R}_{N}$ of rational $f$ of the form

$$
f(\lambda)=a \lambda+b-\sum_{k=1}^{N} \frac{b_{k}}{\lambda-c_{k}}
$$

admitting a rather rich spectral theory. In fact these $f$ will also belong to a class usually associated with the names of Herglotz or Nevanlinna. Boundary-value problems as above involving functions $f$ of this (and more-general) type have been analysed in, for example, $[\mathbf{1 0}, \mathbf{1 1}, \mathbf{1 3}, \mathbf{2 4}]$. These papers (and those cited earlier) have focused mainly on operator-theoretic formulations in Hilbert, Pontryagin or Krein spaces, usually leading to expansion theorems (see also the second part of our study, where further attention to this aspect will be given). Here we shall use differential equation techniques to derive properties of the eigenvalues and eigenfunctions generalizing classical Sturm theory.

A key tool in our analysis will be a modification of a transformation which was first used to our knowledge by Darboux (see [18, p. 132]), and which has subsequently been explored in $[\mathbf{1}, \mathbf{7}, \mathbf{8}]$. These papers focus on transformation of the differential equation $(1.1)$, but for us the effect on the boundary conditions will be crucial. Moreover, if an original boundary condition is Dirichlet, then the cited works produce a transformed problem which is singular, whereas we ensure regularity. This is an important difference since non-Dirichlet conditions transform to Dirichlet, and repeated transformations will be needed. It turns out that each class $\mathcal{R}_{N}$ is the union of two subclasses $\mathcal{R}_{N}^{+}$and $\mathcal{R}_{N}^{0}$ and our transformation will provide direct links between these subclasses for various values of $N$. This fact and the connections with Herglotz-Nevanlinna functions are derived in $\S 2$.

Section 3 contains an analysis of existence, oscillation and comparison theory, mainly via Prüfer methods. In contrast with the usual Sturm theorem, which gives one eigenvalue per oscillation count, here $N$ 'extra' eigenvalues appear with arbitrary oscillation counts (for example, all could be equal). In $\S 4$ we show that if the transformation of the previous paragraph is applied to (1.1)-(1.3), then the new spectrum contains the old eigenvalues (except possibly the first one). Using the oscillation theory of $\S 3$, we show that these are the only eigenvalues of the new problem, so the transformation is isospectral (with the exception above). The new problem is 'simpler' than the original one, and after at most $2 N+1$ transformations we eventually produce a standard problem, i.e. with constant boundary conditions (Corollary 4.2). In $\S 5$, we discuss eigenvalue asymptotics, again via repeated transformations. An alternative approach, based on the 'asymptotic problem' given in $[4]$ for a special case, is also considered. 


\section{Preliminaries}

We consider the class $\mathcal{R}_{N}$ of rational functions $f$ as in (1.4) where all the coefficients are real and $a \geqslant 0, b_{k}>0$ and $c_{1}<c_{2}<\cdots<c_{N}, N \geqslant 0$.

Recall that $f: \mathbb{C} \rightarrow \mathbb{C}$ is a Herglotz-Nevanlinna function if $f(\bar{z})=\overline{f(z)}$ and $f$ maps the closed upper half-plane into itself.

Lemma 2.1. A rational function $f$ with simple real poles is a Herglotz-Nevanlinna function if and only if $f \in \mathcal{R}_{N}$ for some $N$.

Proof. If $f \in \mathcal{R}_{N}$, then each summand is evidently Herglotz-Nevanlinna, and hence so is $f$. Conversely, simplicity of the poles allows a partial fraction expansion of the form

$$
f(\lambda)=p(\lambda)+a \lambda+b-\sum_{k=1}^{N} \frac{b_{k}}{\lambda-c_{k}},
$$

where $p$ is a polynomial whose terms all have degree at least two, and the remaining summands contain coefficients unrestricted in sign. Since $f$ maps the upper half-plane into itself, we can let $\lambda$ tend to $\infty$ along appropriate rays to see that $p$ must vanish and $a$ must be positive. Finally, letting $\lambda$ tend to $c_{k}$ we obtain positivity of $b_{k}$.

Let $\mathcal{R}_{N}^{+}$(respectively, $\mathcal{R}_{N}^{0}$ ) denote the subclass of $\mathcal{R}_{N}$ for which $a>0$ (respectively, $a=0$ ). The following properties are easily established.

Lemma 2.2. Let $f \in \mathcal{R}_{N}$. Then

(i) $f^{\prime}(\lambda)>0$ for each real $\lambda$, where $f(\lambda)$ is finite;

(ii) $\lim _{\lambda \rightarrow c_{k} \pm} f(\lambda)=\mp \infty$; and

(iii) if $f \in \mathcal{R}_{N}^{+}$, then $\lim _{\lambda \rightarrow \pm \infty} f(\lambda)= \pm \infty$, while if $f \in \mathcal{R}_{N}^{0}$, then $f(\lambda) \rightarrow b$ from below (respectively, above) as $\lambda \rightarrow \infty$ (respectively, $-\infty$ ).

The graph of a typical member of $\mathcal{R}_{N}$ is shown in Figure 1.

Given a function $f \in \mathcal{R}_{N}$, and a constant $\mu<c_{1}$, we define

$$
F(\lambda)=\frac{\mu-\lambda}{f(\lambda)-f(\mu)}-f(\mu)
$$

extending this definition by continuity where possible, so $F\left(c_{k}\right)=-f(\mu), 1 \leqslant k \leqslant N$, and $F(\mu)=-f^{\prime}(\mu)^{-1}-f(\mu)$. The principal result of this section shows, in particular, that if $f \in \mathcal{R}_{N}$, then $F \in \mathcal{R}_{M}$, i.e.

$$
F(\lambda)=A \lambda+B-\sum_{k=1}^{M} \frac{B_{k}}{\lambda-C_{k}},
$$

where $M$ is $N-1$ or $N$ depending on the value of $a$. 


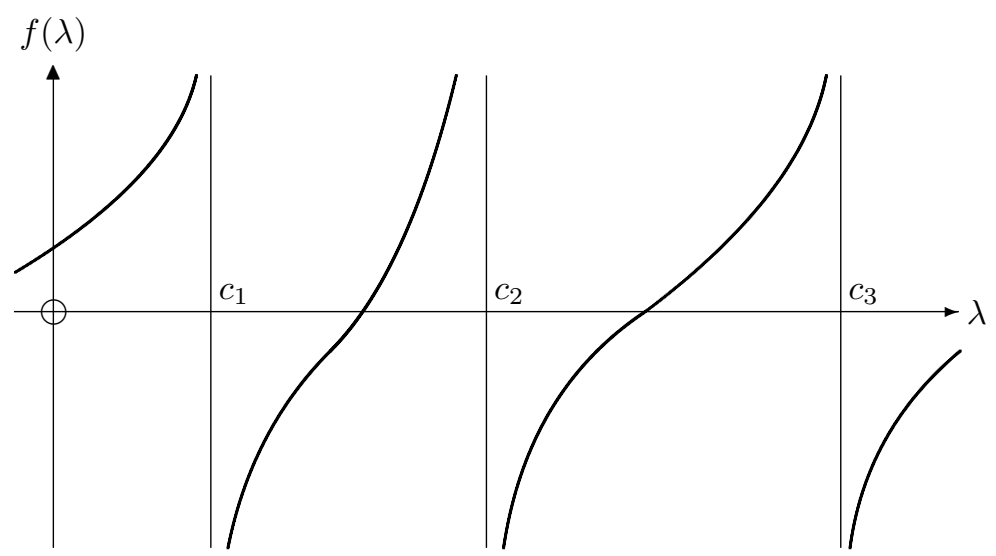

Figure 1. $f(\lambda)$.

Theorem 2.3. In the notation above,

(i) if $f \in \mathcal{R}_{N}^{+}$, then $F \in \mathcal{R}_{N}^{0}$ and $\mu<c_{1}<C_{1}<c_{2}<\cdots<c_{N}<C_{N}$; and

(ii) if $f \in \mathcal{R}_{N}^{0}$, then $F \in \mathcal{R}_{N-1}^{+}$and $\mu<c_{1}<C_{1}<c_{2}<\cdots<C_{N-1}<c_{N}$.

Proof. We calculate

$$
\begin{aligned}
F(\lambda) & =-\frac{\prod_{k=1}^{N}\left(\lambda-c_{k}\right)}{r(\lambda)}-f(\mu) \\
& =\frac{p(\lambda)}{r(\lambda)},
\end{aligned}
$$

where $p(\lambda)$ is a polynomial of degree at most $N$,

$$
r(\lambda)=a \prod_{k=1}^{N}\left(\lambda-c_{k}\right)+\sum_{k=1}^{N} e_{k} \prod_{j=1, j \neq k}^{N}\left(\lambda-c_{j}\right)
$$

is a polynomial of degree $N$ (respectively, $N-1$ ) if $a>0$ (respectively, $a=0$ ) and $e_{k}=b_{k} /\left(\mu-c_{k}\right)<0$.

It is easy to check that

$$
\operatorname{sgn}\left(r\left(c_{k}\right)\right)=(-1)^{N-k+1}, \quad k=1, \ldots, N,
$$

and thus $r$ has roots $\mu_{k} \in\left(c_{k}, c_{k+1}\right), k=1, \ldots, N-1$. When $a=0$, these must be all the roots of $r$ since its degree is $N-1$. When $a>0$, we see that $r\left(c_{N}\right)<0$ and that $r$ has $a>0$ as its leading coefficient. Hence there is an additional root $\mu_{N}>c_{N}$, and now the $\mu_{k}, k=1, \ldots, N$, form all the roots of $r$. All of these roots are simple so the rational function $F$ has simple poles comprising $\mu, \mu_{k}, k=1, \ldots, N$, interlacing the poles of $f$ in the manner described. 
From this it readily follows that $F$ can be expressed in the form (2.2) where $A=0$ and $M=N$ when $a>0$, and, when $a=0, M=N-1$ and $A=-\left(\sum_{k=1}^{N} e_{k}\right)^{-1}>0$ by (2.5). The poles of $F$ are precisely the roots of $f(\lambda)=f(\mu)$ (except $\lambda=\mu$ ), so the basic properties of $f$ show that

$$
f(\lambda)-f(\mu) \rightarrow 0 \pm \quad \text { as } \lambda \rightarrow C_{k} \pm
$$

Thus the original definition of $F$ gives

$$
\lim _{\lambda \rightarrow C_{k} \pm} F(\lambda)=\mp \infty
$$

which forces $B_{k}>0, k=1, \ldots, M$.

Remark 2.4. By composing these transformations we can map $\mathcal{R}_{N}$ into $\mathcal{R}_{0}^{0}$, which will enable us to convert eigenvalue problems with boundary conditions in $\mathcal{R}_{N}$ into standard problems with boundary conditions independent of $\lambda$.

Remark 2.5. Routine algebraic calculations can be used to give $A, B$, etc., in terms of $a, b$, etc. For example,

$$
\begin{aligned}
& f \in \mathcal{R}_{N}^{+} \quad \Longrightarrow A=0, \quad B=-\frac{1}{a}-f(\mu), \\
& f \in \mathcal{R}_{N}^{0} \quad \Longrightarrow A=-\left(\sum_{k=1}^{N} e_{k}\right)^{-1}
\end{aligned}
$$

\section{Eigenvalues: existence and oscillation theory}

We consider here the existence of eigenvalues and the associated oscillation theory for the problem (1.1)-(1.3) where $f \in \mathcal{R}_{N}$. If $\alpha=0$, we interpret (1.2) as $y(0)=0$. Our approach will be via Prüfer theory, and to this end for a given $\lambda$ we consider the solution $y(\lambda, x)$ of $(1.1),(1.2)$ and define $\theta(\lambda, x)$ via the initial-value problem

$$
\theta^{\prime}=\cos ^{2} \theta+(\lambda-q) \sin ^{2} \theta, \quad \theta(\lambda, 0)=\alpha,
$$

which leads to

$$
\cot \theta(\lambda, x)=\frac{y^{\prime}(\lambda, x)}{y(\lambda, x)}
$$

In particular the eigencondition (1.3) becomes

$$
\cot \theta(\lambda, 1)=f(\lambda)
$$

Standard properties of the Prüfer angle $\theta$ (for example, that for a given $x, \theta(\lambda, x)$ is continuous and increasing in $\lambda$, and that $\lim _{\lambda \rightarrow-\infty} \theta(\lambda, 1)=0$ and $\left.\lim _{\lambda \rightarrow+\infty} \theta(\lambda, 1)=\infty\right)$ can be found in, for example, $[\mathbf{2}]$. The graph of $\cot \theta(\lambda, 1)$ is displayed in Figure 2.

Geometrically, the real eigenvalues of (1.1)-(1.3) correspond to the $\lambda$-values at which the graphs of $\cot \theta(\lambda, 1)$ and $f(\lambda)$ intersect. (If these graphs share a common vertical 


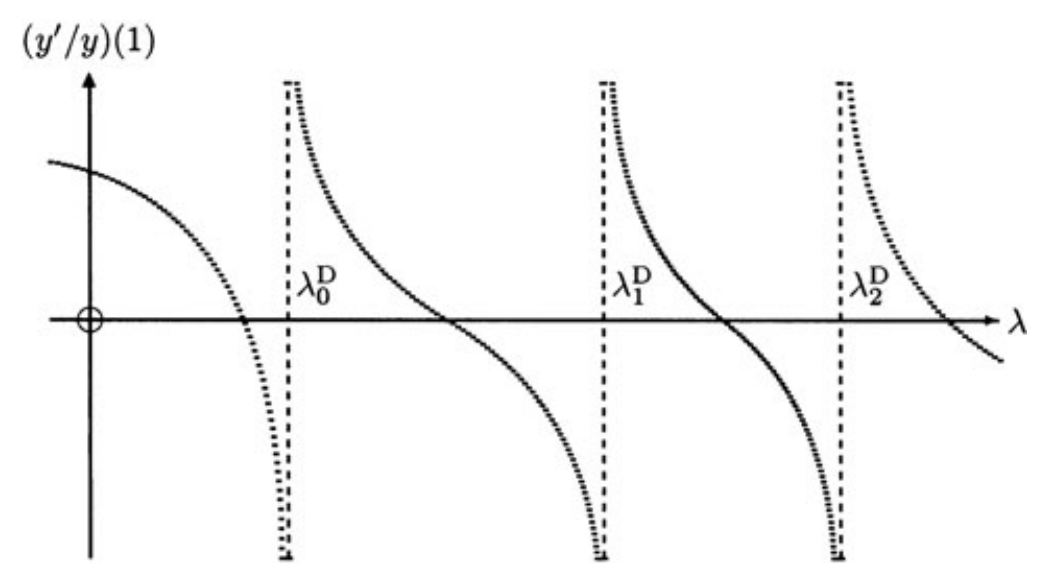

Figure 2. $\cot \theta(\lambda, 1)$.

asymptote $c_{k}$, say, then $\lambda=c_{k}$ is an eigenvalue for the terminal condition $y(1)=0$.) Since $\cot \theta(\lambda, 1)$ and $f(\lambda)$ are, respectively, decreasing and increasing on each branch, there is a sequence of simple intersections of these two graphs.

More precisely, we define $y(x, \lambda)$ to be a non-zero solution of $(1.1),(1.2)$, analytic in $\lambda \in \mathbb{C}$, and we write $\omega(\lambda)=y^{\prime}(1, \lambda)-f(\lambda) y(1, \lambda)$. By definition, $\lambda$ is an eigenvalue of (1.1)-(1.3) if $\omega(\lambda)=0$, and we call $\lambda$ a simple eigenvalue if, in addition, $\omega_{\lambda}(\lambda) \neq 0$, where the suffix denotes differentiation with respect to $\lambda$. To discuss the poles of $f$ we use $\Omega(\lambda)=y(1, \lambda)-y^{\prime}(1, \lambda) / f(\lambda)$ instead of $\omega(\lambda)$. Specifically, $\lambda=c_{k}$ is an eigenvalue if $y(1, \lambda)=0$, and is a simple eigenvalue if, in addition, $y_{\lambda}(1, \lambda) \neq 0$.

\section{Theorem 3.1.}

(i) The eigenvalues of (1.1)-(1.3) are real, simple and form a sequence $\lambda_{0}<\lambda_{1}<\cdots$ accumulating only at $\infty$ and with $\lambda_{0}<c_{1}$.

(ii) If $b$ is decreased and $c_{k}, q$ are increased, then each $\lambda_{j}$ is increased.

(iii) If $a>0$ is decreased and $b_{k}$ is increased, then each positive $\lambda_{j}>c_{k}$ is increased.

Proof. (i) Suppose $\lambda$ is a non-real eigenvalue. Then (1.1)-(1.3) hold with $y \neq 0$, and also

$$
\begin{aligned}
-\bar{y}^{\prime \prime}+q \bar{y} & =\overline{\lambda y} \\
\left(\bar{y}^{\prime} / \bar{y}\right)(0) & =\cot \alpha
\end{aligned}
$$

and

$$
\left(\bar{y}^{\prime} / \bar{y}\right)(1)=\overline{f(\lambda)}=f(\bar{\lambda}),
$$

since $f$ is Herglotz-Nevanlinna. Thus $\bar{\lambda}$ is also an eigenvalue and without loss we shall assume $\operatorname{Im} \lambda>0$. 
Now by the standard procedure of multiplying (1.1) by $\bar{y},(3.3)$ by $y$, integrating and subtracting, we obtain

$$
\left(-y^{\prime} \bar{y}+y \bar{y}^{\prime}\right)(1)=(\lambda-\bar{\lambda}) \int_{0}^{1}|y|^{2},
$$

which we shall call Lagrange's formula for (1.1) and (3.3). The right-hand side is positive imaginary but the left-hand side is $(\overline{f(\lambda)}-f(\lambda))|y(1)|^{2}$, which is negative imaginary since $f$ is Herglotz-Nevanlinna. This contradiction establishes reality of the eigenvalues.

To prove simplicity, suppose $\omega(\lambda)=\omega_{\lambda}(\lambda)=0$ for some $\lambda$, which by the above reasoning we take to be real, and hence without loss of generality we also assume $y$ to be real. From (1.1)-(1.3) we obtain

$$
\begin{aligned}
-y_{\lambda}^{\prime \prime}+q y_{\lambda} & =\lambda y_{\lambda}+y \\
\left(y_{\lambda}^{\prime} / y_{\lambda}\right)(0) & =\cot \alpha
\end{aligned}
$$

and

$$
\frac{y_{\lambda}^{\prime}}{y}(1)=f^{\prime}(\lambda)+f(\lambda) \frac{y_{\lambda}}{y}(1)
$$

By Lagrange's formula for (1.1) and (3.4) we have

$$
\left(-y^{\prime} y_{\lambda}+y y_{\lambda}^{\prime}\right)(1)=-\int_{0}^{1} y^{2} .
$$

The right-hand side is negative, whereas by (3.5) the left-hand side is

$$
y(1)^{2}\left[-f(\lambda) \frac{y_{\lambda}}{y}(1)+f^{\prime}(\lambda)+f(\lambda) \frac{y_{\lambda}}{y}(1)\right]=y(1)^{2} f^{\prime}(\lambda),
$$

which is positive by Lemma $2.2(\mathrm{i})$, and we obtain a contradiction. If $\lambda=c_{k}$ is a nonsimple eigenvalue, then $y\left(c_{k}\right)=y_{\lambda}\left(1, c_{k}\right)=0$, so the left-hand side of (3.6) vanishes but the right-hand side is negative, and again we have a contradiction.

The final contention follows from the geometry of the $\cot \theta$ and $f$ graphs.

(ii) We note that a decrease in $b$ or an increase in any of $c_{k}, 1 \leqslant k \leqslant N$, causes $f(\lambda)$ to decrease, while an increase in $q$ causes $\theta(\lambda, 1)$ to increase (see (3.2)). The net effect is an increase in each $\lambda_{j}$. The proof of (iii) is similar.

Closer examination of the graphs reveals some interesting interlacing relationships which we shall now explore. We define $\lambda_{i}^{\mathrm{D}}, i=0,1, \ldots$, to be the eigenvalues for the standard Sturm-Liouville problem consisting of (1.1)-(1.2) and the Dirichlet condition $y(1)=0$. We also use the notation $\lambda_{i}^{c \mathrm{D}}$ to denote the sequence consisting of all $c_{j}$ and $\lambda_{k}^{\mathrm{D}}$ in non-decreasing order (counted by multiplicity if $c_{j}=\lambda_{k}^{\mathrm{D}}$ for some $j$ and $k$ ). Let $k_{j}$ be the number of points $c_{i} \leqslant \lambda_{j}$. 


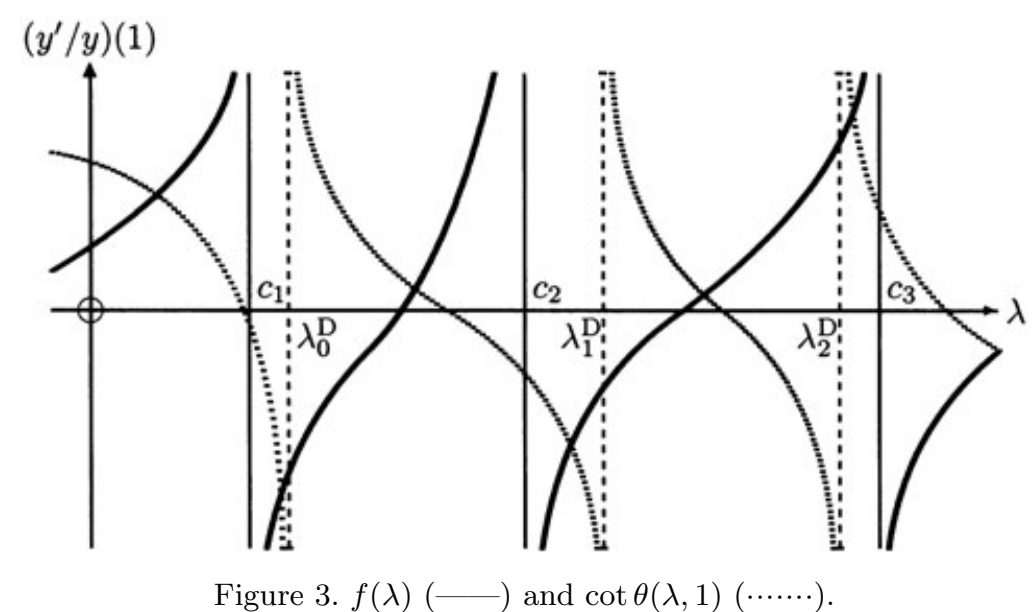

Analysis of the $f$ and $\cot \theta$ graphs (cf. Figure 3) now yields the following theorem.

Theorem 3.2.

(i) The $\lambda_{i}$ interlace the $\lambda_{j}^{c \mathrm{D}}$ in the sense that $\lambda_{0}<\lambda_{0}^{c \mathrm{D}} \leqslant \lambda_{1} \leqslant \lambda_{1}^{c \mathrm{D}} \leqslant \cdots$.

(i) Where defined, $c_{k_{j}} \leqslant \lambda_{j}<c_{k_{j}+1}$.

(iii) Setting $\lambda_{-1}^{\mathrm{D}}=-\infty$ we have $\lambda_{j-1-k_{j}}^{\mathrm{D}}<\lambda_{j} \leqslant \lambda_{j-k_{j}}^{\mathrm{D}}$, for all $j \geqslant 0$.

We can now deduce the oscillation properties of the eigenfunctions. Note that if $\lambda \in$ $\left(\lambda_{i-1}^{\mathrm{D}}, \lambda_{i}^{\mathrm{D}}\right]$, then a solution of $(1.1)-(1.2)$ has $i$ zeros in $(0,1)$. Thus the oscillation number (i.e. the number of zeros in $(0,1)$ of an eigenfunction) associated with such an eigenvalue can be found by determining the interval $\left(\lambda_{i-1}^{\mathrm{D}}, \lambda_{i}^{\mathrm{D}}\right]$ into which it falls.

Corollary 3.3. Let $\omega_{j}$ be the oscillation number associated with $\lambda_{j}$. Then $\omega_{j}=j-k_{j}$ and, in particular, $\omega_{0}=0$ and $\omega_{j}=j-N$ when $\lambda_{j}>c_{N}$.

\section{Transformations between problems}

The aim of this section is to describe a transformation, with certain eigenvalue-preserving properties, from a problem of the type (1.1)-(1.3) to a 'simpler' one with a new potential $\hat{q}$ in place of $q$ and with $f(\lambda)$ replaced by $F(\lambda)$ constructed in $\S 2$. In this context 'simpler' means that $F$ has either fewer terms or fewer poles than $f$. By iteration of the procedure one can transform (1.1)-(1.3) to a standard Sturm-Liouville problem whose eigenvalues are those of the original problem except for finitely many. For brevity we denote the oscillation number of a function $y$ on $(0,1)$ by $\operatorname{osc}(y)$.

Theorem 4.1. For the problem (1.1)-(1.3) suppose the eigenvalues are $\lambda_{0}<\lambda_{1}<\cdots$ with corresponding eigenfunctions $y_{0}, y_{1}, \ldots$ Let

$$
\begin{aligned}
& \mu=\lambda_{0} \quad \text { if } \alpha>0, \\
& \mu<\lambda_{0} \quad \text { if } \alpha=0,
\end{aligned}
$$


and let $w$ be the solution of (1.1) with $\lambda=\mu, w(1)=1$ and $w^{\prime}(1)=f(\mu)$. Define

$$
z=\left(w^{\prime} / w\right) \text { and } \hat{q}=q-2 z^{\prime} .
$$

Let $F(\lambda)$ be as in (2.1) and define $\gamma \in[0, \pi)$ by $\cot \gamma=-z(0)$ if $\alpha=0$ and $\gamma=0$ if $\alpha>0$. Then the eigenvalues of the problem

$$
\begin{aligned}
-y^{\prime \prime}+\hat{q} y & =\lambda y, \\
\left(y^{\prime} / y\right)(0) & =\cot \gamma, \\
\left(y^{\prime} / y\right)(1) & =F(\lambda)
\end{aligned}
$$

are $\lambda_{j}$, with corresponding eigenfunctions $u_{j}=y_{j}^{\prime}-z y_{j}$, where $j \geqslant 1$ for $\alpha>0$ and $j \geqslant 0$ for $\alpha=0$.

Proof. We begin by showing that $w$ has no zeros in $[0,1]$. This is clear when $\alpha>0$, for then $w=y_{0}$, which has no zeros in $(0,1)$, and, moreover, $\left(w^{\prime} / w\right)(0)=\cot \alpha$ and $\left(w^{\prime} / w\right)(1)=f\left(\lambda_{0}\right)$, both of which are finite.

When $\alpha=0$, we note that $y_{0}(0)=0$ but $y_{0}$ has no zeros in $(0,1)$ and $y_{0}(1) \neq 0$ since $\lambda_{0}<\lambda_{0}^{\mathrm{D}}$. Now consider the Prüfer differential equation (3.1) with $\lambda=\mu<\lambda_{0}$ and subject to the terminal condition $\theta(\mu, 1)=\cot ^{-1} f\left(\lambda_{0}\right) \in(0, \pi)$. Since $\mu<\lambda_{0}$, we obtain a positive initial value $\theta(\mu, 0) \in(0, \pi)$. (Recall that $\theta(\mu, x)$ cannot decrease through multiples of $\pi$ as $x$ decreases.) If we now replace the terminal condition by $\theta(\mu, 1)=\cot ^{-1} f(\mu)>\cot ^{-1} f\left(\lambda_{0}\right)$, we see that $\theta(\mu, 0)$ increases again but must remain within $(0, \pi)$ provided we choose the terminal angle to be less than $\pi$.

Thus the solution $\psi(x)$ of the Prüfer equation with $\lambda=\mu$ and terminal condition $\psi(1)=\cot ^{-1} f(\mu)$ takes values only in $(0, \pi)$ for all $x \in[0,1]$. This establishes that $w$ has no zeros in $[0,1]$, and consequently $z$ and $\gamma$ as given in the statement are well defined.

For the case $\alpha>0$ we shall define $\psi(x)=\theta\left(\lambda_{0}, x\right)$. Thus

$$
\psi(0)= \begin{cases}\alpha & \alpha>0, \\ \pi-\gamma & \alpha=0 .\end{cases}
$$

Throughout the proof, when we refer to $j$, we mean $j \geqslant 1$ if $\alpha>0$ and $j \geqslant 0$ for $\alpha=0$. As in [6], direct calculation shows that the functions $u_{j}$ satisfy (4.1) and (4.2) and further that

$$
\frac{u_{j}^{\prime}}{u_{j}}=\frac{\mu-\lambda_{j}}{\left(y_{j}^{\prime} / y_{j}\right)-z}-z \quad \text { on }[0,1]
$$

so $u_{j}$ also satisfies (4.3). Thus the $\lambda_{j}$ are indeed eigenvalues for (4.1)-(4.3) and it remains to show that they constitute all the eigenvalues.

Let $\phi(\lambda, x)$ be the Prüfer angle generated by (4.1) with $\phi(\lambda, 0)=\gamma$ as in (4.2). In a similar manner let $\theta(\lambda, x)$ be generated by (1.1) with $\theta(\lambda, 0)=\alpha$ as in (1.2). Note that

$$
\frac{u_{j}^{\prime}}{u_{j}}(x)=\cot \phi\left(\lambda_{j}, x\right), \quad \frac{y_{j}^{\prime}}{y_{j}}(x)=\cot \theta\left(\lambda_{j}, x\right)
$$


and $z(x)=\cot \psi(x)$. Thus we see from (4.4) that

$$
\cot \phi\left(\lambda_{j}, x\right)=\frac{\mu-\lambda_{j}}{\cot \theta\left(\lambda_{j}, x\right)-\cot \psi(x)}-\cot \psi(x) .
$$

Recall that $\psi(x) \in(0, \pi)$ for all $x \in[0,1]$. Since $\lambda_{j}>\mu$ when $j \geqslant 1$, we have $\theta\left(\lambda_{j}, x\right)>$ $\psi(x)$ on $(0,1]$ if $\alpha>0$, while $0=\theta\left(\lambda_{j}, 0\right)<\psi(0)$ for $\alpha=0$.

Further, we see from the Prüfer equation for $\theta$ that if $\cot \theta\left(\lambda_{j}, x\right)=\cot \psi(x)$ for some $x$, then $(\mathrm{d} / \mathrm{d} x)\left(\theta\left(\lambda_{j}, x\right)-\psi(x)\right)>0$ from (3.1), since $\sin ^{2} \psi(x)>0$. Thus $\theta\left(\lambda_{j}, x\right)-\psi(x)$ increases through multiples of $\pi$. From (4.4) it follows that $u_{j}$ has a zero at $x \in(0,1)$ (or, equivalently, $\cot \phi\left(\lambda_{j}, x\right)$ is undefined) if and only if $\theta\left(\lambda_{j}, x\right)=\psi(x)+m \pi$ for some positive (respectively, non-negative) integer $m$, for $\alpha>0$ (respectively, $\alpha=0$ ). Thus, since $\phi\left(\lambda_{j}, x\right)$ increases through multiples of $\pi, \operatorname{osc}\left(u_{j}\right)=n$ if and only if

$$
\begin{array}{cc}
n \pi<\theta\left(\lambda_{j}, 1\right)-\psi(1) \leqslant(n+1) \pi & \text { if } \alpha>0, \\
(n-1) \pi<\theta\left(\lambda_{j}, 1\right)-\psi(1) \leqslant n \pi & \text { if } \alpha=0 .
\end{array}
$$

By Theorem $3.2(\mathrm{iii}), \lambda_{j} \in\left(\lambda_{i-1}^{\mathrm{D}}, \lambda_{i}^{\mathrm{D}}\right]$, where $i=j-k_{j}$ and so $i \pi<\theta\left(\lambda_{j}, 1\right) \leqslant(i+1) \pi$, from which we obtain $(i-1) \pi<\theta\left(\lambda_{j}, 1\right)-\psi(1)<(i+1) \pi$. Thus $\operatorname{osc}\left(u_{j}\right)$ is $i-1$ or $i$, for $\alpha>0$, and $i$ or $i+1$, for $\alpha=0$, according to whether $\theta\left(\lambda_{j}, 1\right)-\psi(1) \leqslant i \pi$ or $\theta\left(\lambda_{j}, 1\right)-\psi(1)>i \pi$. In terms of $f$ and $\cot \theta(\lambda, 1)$ (recall that $f(\mu)=z(1)=\cot \psi(1)$ ), we see that $\operatorname{osc}\left(u_{j}\right)$ is $i-1$ or $i$, for $\alpha>0$, and $i$ or $i+1$, for $\alpha=0$, according to whether $f(\mu) \leqslant \cot \theta\left(\lambda_{j}, 1\right)$ or $f(\mu)>\cot \theta\left(\lambda_{j}, 1\right)$.

We recall the expressions for $f(\lambda)$ and $F(\lambda)$ from $\S 2$ :

$$
f(\lambda)=a \lambda+b-\sum_{k=1}^{N} \frac{b_{k}}{\lambda-c_{k}}, \quad F(\lambda)=A \lambda+B-\sum_{k=1}^{M} \frac{B_{k}}{\lambda-C_{k}},
$$

where $A=0, M=N$ if $a>0$ and $A>0, M=N-1$ if $a=0$. Recall also that the $C_{k}$ are the solutions other than $\lambda=\mu$ of $f(\lambda)=f(\mu)$.

Now when $a>0$, we have for $j$ large enough to ensure $\lambda_{j}>C_{N}>c_{N}$,

$$
\left(y_{j}^{\prime} / y_{j}\right)(1)=\cot \theta\left(\lambda_{j}, 1\right)=f\left(\lambda_{j}\right)>f\left(C_{N}\right)=f(\mu),
$$

so $\operatorname{osc}\left(u_{j}\right)=\operatorname{osc}\left(y_{j}\right)-1$, which equals $j-N-1$ by Corollary 3.3, for $\alpha>0$. Similarly for $\alpha=0, \operatorname{osc}\left(u_{j}\right)=\operatorname{osc}\left(y_{j}\right)=j-N$. On the other hand, if we regard $\lambda_{j}$ as the $k$ th eigenvalue $(k \geqslant 0)$ of $(4.1)-(4.3)$ listed in increasing order, Corollary 3.3 shows that the oscillation number associated with $\lambda_{j}$ is $k-N$, so $k=j-1$ for $\alpha>0$, and $k=j$ for $\alpha=0$. Hence the $\lambda_{j}, j \geqslant 1$ for $\alpha>0$ and $j \geqslant 0$ for $\alpha=0$, constitute all the eigenvalues of (4.1)-(4.3).

When $a=0$, we have for $j$ large enough to ensure $\lambda_{j}>c_{N}$,

$$
\left(y_{j}^{\prime} / y_{j}\right)(1)=\cot \theta\left(\lambda_{j}, 1\right)=f\left(\lambda_{j}\right)<b<f(\mu),
$$

showing that $\operatorname{osc}\left(u_{j}\right)=\operatorname{osc}\left(y_{j}\right)=j-N$, for $\alpha>0$, while for $\alpha=0, \operatorname{osc}\left(u_{j}\right)=\operatorname{osc}\left(y_{j}\right)+1=$ $j-N+1$. The argument concludes as before, since in this case $M=N-1$. 
By iterating these constructions, we arrive at the following corollary.

Corollary 4.2. Given (1.1)-(1.3) with $f \in \mathcal{R}_{N}$, there exists a Sturm-Liouville problem $-y^{\prime \prime}+q_{0} y=\mu y$ subject to $\left(y^{\prime} / y\right)(j)=\cot \alpha_{j}, j=0,1$, with eigenvalues $\mu_{0}<\mu_{1}<$ $\cdots$, where

(i) $\alpha_{0}=0$ and $\mu_{j}=\lambda_{j+N+1}$ if $\alpha>0, f \in \mathcal{R}_{N}^{+}$;

(ii) $\alpha_{0}=0$ and $\mu_{j}=\lambda_{j+N}$ if $\alpha=0, f \in \mathcal{R}_{N}^{0}$; and

(iii) $\alpha_{0}>0$ and $\mu_{j}=\lambda_{j+N}$ otherwise.

\section{Asymptotics of eigenvalues}

Our aim in this section is to show how eigenvalue asymptotics for the problem (1.1)(1.3) can be derived from those for 'standard' Sturm-Liouville problems (with constant boundary conditions). It is well known that asymptotics for the latter are available to any order, depending on the smoothness of $q$ (cf. [20, p. 23]). For $q \in L^{1}$, we have the following well-known estimates (see, for example, [4, Theorem A3]).

Theorem 5.1. If $\lambda_{j}^{\prime}$ denote the eigenvalues of the problem (1.1) with $\rho=y^{\prime} / y$ constant at 0 and 1 , then

$$
\lambda_{j}^{\prime}=j^{2} \pi^{2}+o\left(j^{2}\right)
$$

as $j \rightarrow \infty$. In fact,

$$
\lambda_{j}^{\prime}=(j+D)^{2} \pi^{2}+\int_{0}^{1} q-2\left[\rho^{*}\right]_{0}^{1}+o(1 / j),
$$

where $D$ is half the number of Dirichlet conditions specified, $\rho^{*}=\rho$ if $\rho$ is finite, and $\rho^{*}=0$ otherwise.

Successive applications of the transformations of $\S 4$, as in Corollary 4.2 , lead to the following corollary.

Corollary 5.2. For the problem (1.1)-(1.3), (5.1) holds with $\lambda_{j}^{\prime}$ replaced by $\lambda_{j}$.

Higher-order asymptotics may be obtained via the same technique, although since one has to keep track of the boundary terms at each stage, an inductive argument is appropriate as below.

\section{Theorem 5.3.}

(i) If $f \in \mathcal{R}_{N}^{0}$, then, as $j \rightarrow \infty$,

$$
\lambda_{j}= \begin{cases}(j-N)^{2} \pi^{2}+\int_{0}^{1} q-2 b+2 \cot \alpha+o(1 / j), & \alpha \neq 0, \\ \left(j+\frac{1}{2}-N\right)^{2} \pi^{2}+\int_{0}^{1} q-2 b+o(1 / j), & \alpha=0 .\end{cases}
$$


(ii) If $f \in \mathcal{R}_{N}^{+}$, then, as $j \rightarrow \infty$,

$$
\lambda_{j}= \begin{cases}\left(j-\frac{1}{2}-N\right)^{2} \pi^{2}+\int_{0}^{1} q+2 \cot \alpha+(2 / a)+o(1 / j), & \alpha \neq 0, \\ (j-N)^{2} \pi^{2}+\int_{0}^{1} q+(2 / a)+o(1 / j), & \alpha=0 .\end{cases}
$$

Proof. When $N=0$, (i) and (ii) follow from Theorem 5.1 and [4], so we can assume that (i) and (ii) hold for $0,1, \ldots, N$.

(i) Suppose $\alpha>0$ and $f \in \mathcal{R}_{N+1}^{0}$. Then $F \in \mathcal{R}_{N}^{+}$and denoting the other transformed quantities by carets, we have $\hat{q}=q-2 z^{\prime}$ and $\hat{\alpha}=0$. By (5.3) and the inductive hypothesis,

$$
\lambda_{j+1}=(j-N)^{2} \pi^{2}+\int_{0}^{1} \hat{q}+(2 / \hat{a})+o(1 / j)
$$

so by $(2.7)$

$$
\begin{aligned}
\lambda_{j} & =(j-(N+1))^{2} \pi^{2}+\int_{0}^{1} q-2\left[f\left(\lambda_{0}\right)-\cot \alpha+\Sigma e_{k}\right]+o(1 / j) \\
& =(j-(N+1))^{2} \pi^{2}+\int_{0}^{1} q-2 b+2 \cot \alpha+o(1 / j)
\end{aligned}
$$

as $\mu=\lambda_{0}$. This establishes (5.2) for $N+1$ and $\alpha>0$.

Now suppose $\alpha=0$ instead. Then $\hat{\alpha}>0$ and we use (5.3) and (2.7) to obtain

$$
\begin{aligned}
\lambda_{j} & =\left(j-\frac{1}{2}-N\right)^{2} \pi^{2}+\int_{0}^{1} \hat{q}+2 \cot \hat{\alpha}+(2 / \hat{a})+o(1 / j) \\
& =\left(j+\frac{1}{2}-(N+1)\right)^{2} \pi^{2}+\int_{0}^{1} q-2\left[f(\mu)-z(0)+z(0)+\Sigma e_{k}\right]+o(1 / j) \\
& =\left(j+\frac{1}{2}-(N+1)\right)^{2} \pi^{2}+\int_{0}^{1} q-2 b+o(1 / j),
\end{aligned}
$$

which establishes (5.2) for $N+1$ and $\alpha=0$.

(ii) Consider the case when $f \in \mathcal{R}_{N+1}^{+}$. Then $F \in \mathcal{R}_{N+1}^{0}$ and we may apply the results just established in (i). When $\alpha>0$ we have $\hat{\alpha}=0$ and we use (5.2) with $N+1$ in place of $N$ to give

$$
\lambda_{j+1}=\left(j+\frac{1}{2}-(N+1)\right)^{2} \pi^{2}+\int_{0}^{1} \hat{q}-2 \hat{b}+o(1 / j),
$$

so by $(2.6)$

$$
\begin{aligned}
\lambda_{j} & =\left(j-\frac{1}{2}-(N+1)\right)^{2} \pi^{2}+\int_{0}^{1} q-2\left[f\left(\lambda_{0}\right)-\cot \alpha-\left(a^{-1}+f\left(\lambda_{0}\right)\right)\right]+o(1 / j) \\
& =\left(j-\frac{1}{2}-(N+1)\right)^{2} \pi^{2}+\int_{0}^{1} q+2 \cot \alpha+(2 / a)+o(1 / j)
\end{aligned}
$$


thus establishing (5.3) for $N+1$ and $\alpha>0$. Finally, when $\alpha=0$ we use (5.2) and (2.6) to see that

$$
\begin{aligned}
\lambda_{j} & =(j-(N+1))^{2} \pi^{2}+\int_{0}^{1} \hat{q}-2 \hat{b}+2 \cot \hat{\alpha}+o(1 / j) \\
& =(j-(N+1))^{2} \pi^{2}+\int_{0}^{1} q-2\left[f(\mu)-z(0)-\left(a^{-1}+f(\mu)\right)+z(0)\right]+o(1 / j) \\
& =(j-(N+1))^{2} \pi^{2}+\int_{0}^{1} q+(2 / a)+o(1 / j)
\end{aligned}
$$

as in (5.3).

An alternative approach, at least to this order, can be given via a simpler 'asymptotic' problem whose eigenvalues are ultimately close (but not equal) to those of (1.1)-(1.3). We define this asymptotic problem by (1.1), (1.2) and the 'asymptotic boundary condition'

$$
\begin{aligned}
\left(y^{\prime} / y\right)(1)=b & \text { if } f \in \mathcal{R}_{N}^{0}, \\
y(1)=0 & \text { if } f \in \mathcal{R}_{N}^{+} .
\end{aligned}
$$

We label the eigenvalues of this problem by oscillation count as $\lambda_{0}^{\mathrm{A}}<\lambda_{1}^{\mathrm{A}}<\cdots$. The first result concerns the interlacing of the sequences $\lambda_{j}^{\mathrm{A}}, \lambda_{j}^{\mathrm{D}}$ and $\lambda_{j}$.

Theorem 5.4. For the problem (1.1)-(1.3), let $j$ be large enough to ensure $\lambda_{j}>c_{N}$.

(i) If $f \in \mathcal{R}_{N}^{0}$, then $\lambda_{j-N}^{A}<\lambda_{j} \leqslant \lambda_{j-N}^{\mathrm{D}}<\lambda_{j-N+1}^{A}$.

(ii) If $f \in \mathcal{R}_{N}^{+}$, then $\lambda_{j-N-1}^{A}<\lambda_{j}<\lambda_{j-N}^{A}$.

Proof. Part (i) is a simple consequence of the superpositioning of the graphs of $f(\lambda)$ and $\cot \theta(\lambda, 1)$. Part (ii) follows from Theorem $3.2\left(\right.$ iii) and $\lambda_{j}^{\mathrm{A}}=\lambda_{j}^{\mathrm{D}}$.

Sharper estimates involving $\lambda_{j}^{\mathrm{A}}$ and $\lambda_{j}^{\mathrm{D}}$ can be given as follows.

Theorem 5.5. For the problem (1.1)-(1.3),

(i) if $f \in \mathcal{R}_{N}^{0}$, then $\lambda_{j}-\lambda_{j-N}^{A}=O\left(1 / j^{2}\right)$; and

(ii) if $f \in \mathcal{R}_{N}^{+}$, then $\lambda_{j}-\lambda_{j-N-1}^{\mathrm{D}}=(2 / a)+O\left(1 / j^{2}\right)$.

Proof. Let $g(\lambda)=a \lambda+b$ and denote by $\mu_{n}$ the solution in $\left(\lambda_{n-1}^{\mathrm{D}}, \lambda_{n}^{\mathrm{D}}\right)$ of $\cot \theta(\lambda, 1)=$ $g(\lambda)$. Note that $f(\lambda)<g(\lambda)$ for $\lambda \gg 1$, so $\lambda_{n+N}>\mu_{n}$ for $n \gg 1$. We claim that $\lambda_{n+N}-\mu_{n}=O\left(1 / n^{2}\right)$ as $n \rightarrow \infty$. To this end, consider

$$
\begin{aligned}
\cot \theta\left(\lambda_{n+N}, 1\right)-\cot \theta\left(\mu_{n}, 1\right) & =f\left(\lambda_{n+N}\right)-g\left(\mu_{n}\right) \\
& =a\left(\lambda_{n+N}-\mu_{n}\right)+O\left(1 / n^{2}\right)
\end{aligned}
$$


using Theorem 3.2 (iii). From this it follows that, for some $\xi_{n} \in\left(\mu_{n}, \lambda_{n+N}\right)$,

$$
\begin{aligned}
\frac{\mathrm{d}}{\mathrm{d} \lambda} \cot \theta\left(\xi_{n}, 1\right)\left(\lambda_{n+N}-\mu_{n}\right) & =a\left(\lambda_{n+N}-\mu_{n}\right)+O\left(1 / n^{2}\right), \\
\lambda_{n+N}-\mu_{n} & =\frac{O\left(1 / n^{2}\right)}{a-(\mathrm{d} / \mathrm{d} \lambda) \cot \theta\left(\xi_{n}, 1\right)} .
\end{aligned}
$$

Now $(\mathrm{d} / \mathrm{d} \lambda) \cot \theta\left(\xi_{n}, 1\right) \leqslant-\epsilon<0$ is proven in $[\mathbf{4}$, p. 63 , eqn (3.8) and those that follow] and this establishes our claim.

From this, (i) follows directly, and for (ii) we refer to [4, Theorem 5.3], which gives $\mu_{n}-\lambda_{n-1}^{A}=(2 / a)+O\left(n^{-2}\right)$ for the case at hand.

Theorem 5.3 is now a simple consequence of this result and Theorem 5.1. Higher-order asymptotics could in principle be derived by this technique also, but one would need more accurate Taylor expansions of $f(\lambda)$ and $\cot \theta(\lambda, 1)$.

Acknowledgements. The research of P.A.B. and P.J.B. was supported in part by grants from the NSERC of Canada. The research of B.A.W. was conducted while visiting the University of Calgary and the University of Saskatchewan and supported in part by the Centre for Applicable Analysis and Number Theory.

\section{References}

1. V. È. Adler, A modification of Crum's method, Theor. Math. Phys. 101 (1994), 13811386.

2. F. V. AtKinson, Discrete and continuous boundary problems (Academic Press, 1964).

3. A. I. BenedeK And R. PAnzone, On Sturm-Liouville problems with the square root of the eigenvalue parameter contained in the boundary conditions, Notas Algebra Analysis 10 (1981), 1-62.

4. P. A. Binding, P. J. Browne And K. Seddighi, Sturm-Liouville problems with eigenparameter dependent boundary conditions, Proc. Edinb. Math. Soc. 37 (1993), 57-72.

5. P. A. Binding, R. Hryniv, H. Langer and B. Najman, Elliptic eigenvalue problems with eigenparameter dependent boundary conditions, J. Diff. Eqns 174 (2001), 30-54.

6. P. A. Binding, P. J. Browne and B. A. Watson, Transformations between SturmLiouville problems with eigenvalue dependent and independent boundary conditions, Bull. Lond. Math. Soc. 33 (2001), 749-757.

7. M. M. Crum, Associated Sturm-Liouville systems, Q. J. Math. 6 (1955), 121-127.

8. P. DEIFT, Applications of a commutation formula, Duke Math. J. 45 (1978), 267-310.

9. A. DiJKsma, Eigenfunction expansions for a class of J-selfadjoint ordinary differential operators with boundary conditions containing the eigenvalue parameter, Proc. $R$. Soc. Edinb. A 86 (1980), 1-27.

10. A. DiJKsma AND H. LANGER, Operator theory and ordinary differential operators, Fields Inst. Monographs 3 (1996), 75-139.

11. A. Dijksma, H. Langer And H. De Snoo, Symmetric Sturm-Liouville operators with eigenvalue depending boundary conditions, Can. Math. Soc. Conf. Proc. 8 (1987), 87-116.

12. W. Eberhard, G. Freiling And A. Schneider, Note on a paper of E. M. E. Zayed and S. F. M. Ibrahim: 'Eigenfunction expansion for a regular fourth order eigenvalue problem with eigenvalue parameter in the boundary conditions' [Int. J. Math. Math. Sci. 12 (1989), 341-348; MR 90h:34043], Int. J. Math. Math. Sci. 15 (1992), 809-811. 
13. A. E. ÈTKIN, Some boundary value problems with a spectral parameter in the boundary conditions, Am. Math. Soc. Transl. 2136 (1987), 35-41.

14. C. T. Fulton, Two-point boundary value problems with eigenvalue parameter contained in the boundary conditions, Proc. R. Soc. Edinb. A 77 (1977), 293-308.

15. C. T. Fulton, Singular eigenvalue problems with eigenvalue-parameter contained in the boundary conditions, Proc. R. Soc. Edinb. A 87 (1980), 1-34.

16. D. B. Hinton, Eigenfunction expansions for a singular eigenvalue problem with eigenparameter in the boundary conditions, SIAM J. Math. Analysis 12 (1981), 572-584.

17. D. B. Hinton AND J. K. Shaw, Differential operators with spectral parameter incompletely in the boundary conditions, Funkcialaj Ekvacioj 33 (1990), 363-385.

18. E. L. INCE, Ordinary differential equations (Dover, New York, 1956).

19. A. Kozhevnikov AND S. YAKUBOV, On operators generated by elliptic boundary problems with a spectral parameter in boundary conditions, Integ. Eqns Operat. Theory 23 (1995), 205-231.

20. B. M. Levitan and M. G. Gasymov, Determination of a differential equation from two of its spectra, Russ. Math. Surv. 19 (1964), 1-63.

21. E. M. RussakovskiI, Operator treatment of boundary problems with spectral parameters entering via polynomials in the boundary conditions, Funct. Analysis Applic. 9 (1975), 358-359.

22. E. M. Russakovskit, The matrix Sturm-Liouville problem with spectral parameter in the boundary conditions. Algebraic and operator aspects, Trans. Moscow Math. Soc. 57 (1996), 159-184.

23. A. A. Shkalikov, Boundary problems for ordinary differential equations with parameter in the boundary conditions, J. Soviet Math. 33 (1986), 1311-1342.

24. A. V. Štraus, On spectral functions of differential operators, Izv. Akad. Nauk SSSR Ser. Mat. 19 (1955), 201-220.

25. C. TRETTER, On $\lambda$-nonlinear boundary eigenvalue problems, Mathematics Research, vol. 71 (Akademie, 1993).

26. J. WALTER, Regular eigenvalue problems with eigenparameter in the boundary conditions, Math. Z. 133 (1973), 301-312.

27. E. M. E. ZAYED AND S. F. M. IBRAhim, An expansion theorem for an eigenvalue problem on an arbitrary multiply connected domain with an eigenparameter in a general type of boundary conditions, Acta Math. Sinica (NS) 11 (1995), 399-407. 